\title{
Boosting and Balancing Electron and Hole Mobility in Single- and Bi-Layer WSe 2 Devices via Tailored Molecular Functionalization
}

\author{
Marc-Antoine Stoeckel ${ }^{1}$, Marco Gobbi ${ }^{1,2, *}$, Tim Leydecker ${ }^{1}$, Ye Wang ${ }^{1}$, Matilde \\ Eredia $^{1}$, Sara Bonacchi ${ }^{1,3}$, Roberto Verucchi ${ }^{4}$, Melanie Timpel ${ }^{5}$, Marco Vittorio \\ Nardi ${ }^{4,5}$, Emanuele Orgiu, ${ }^{1,6, *}$ and Paolo Samori ${ }^{1, *}$ \\ ${ }^{1}$ Université de Strasbourg, CNRS, ISIS, 67000 Strasbourg, France \\ ${ }^{2}$ Centro de Física de Materiales (CSIC-UPV/EHU), paseo Manuel de Lardizabal 5, E-20018 Donostia, San Sebastián, Spain \\ ${ }^{3}$ Dipartimento di Scienze Chimiche, Università di Padova, Via Marzolo, 1. 35131, Padova. Italia. \\ ${ }^{4}$ Istituto dei Materiali per l'Elettronica ed il Magnetismo, IMEM-CNR, Sezione di Trento, Via alla Cascata 56/C, Povo, \\ 38100 Trento, Italy \\ ${ }^{5}$ Department of Industrial Engineering, University of Trento, Via Sommarive 9, 38123 Trento, Italy \\ ${ }^{6}$ INRS-Centre Énergie Matériaux Télécommunications, 1650 Blv. Lionel-Boulet, J3X 1S2 Varennes, Québec \\ Corresponding authors : samori@unistra.fr; marco gobbi001@ehu.eus; $\underline{\text { emanuele.orgiu@emt.inrs.ca }}$
}

ABSTRACT: $\mathrm{WSe}_{2}$ is a layered ambipolar semiconductor enabling hole and electron transport, which renders it a suitable active component for logic circuitry. However, solid-state devices based on singleand bi-layer $\mathrm{WSe}_{2}$ typically exhibit unipolar transport and poor electrical performances when conventional $\mathrm{SiO}_{2}$ dielectric and $\mathrm{Au}$ electrodes are used. Here, we show that silane-containing functional molecules form ordered monolayers on the top of the $\mathrm{WSe}_{2}$ surface, thereby boosting its electrical performance in single- and bi-layer field-effect transistors. In particular, by employing $\mathrm{SiO}_{2}$ dielectric substrates and top Au electrodes, we measure unipolar mobility as high as $\mu_{\mathrm{h}}=150 \mathrm{~cm}^{2} \mathrm{~V}^{-1} \mathrm{~s}^{-1}$ and $\mu_{\mathrm{e}}=$ $17.9 \mathrm{~cm}^{2} \mathrm{~V}^{-1} \mathrm{~s}^{-1}$ in $\mathrm{WSe}_{2}$ single-layer devices when ad hoc molecular monolayers are chosen. Additionally, by asymmetric double-side functionalization with two different molecules, we provide opposite polarity to the top and bottom layer of bi-layer $\mathrm{WSe}_{2}$, demonstrating nearly balanced ambipolarity at the bi-layer limit. Our results indicate that the controlled functionalization of the two sides of $\mathrm{WSe}_{2}$ mono- and bilayer flakes with highly ordered molecular monolayers offers the possibility to simultaneously achieve energy level engineering and defect functionalization, representing a path towards the deterministic control over charge transport in 2D materials. 
KEYWORDS: $\mathrm{WSe}_{2}$, molecular functionalization, self-assembled monolayers, ambipolar FETs, 2D materials

2D materials (2DMs) are emerging as a potential alternative to silicon technology for next-generation opto- and nano-electronics. ${ }^{1,2}$ While the semi-metallic character of graphene ${ }^{3}$ hampers its application in electronics, transition metal dichalcogenides (TMDs) include semiconducting compounds with a bandgap in the visible range ${ }^{4}$. This class of materials can be exfoliated down to the single layer and are already being employed as active component in high-performance field-effect transistors (FETs). ${ }^{5-7}$

An exemplary case of semiconductive $\mathrm{TMD}$ is $\mathrm{WSe}_{2}$, that holds great potential for complementary digital logic applications ${ }^{8}$ since it can transport both holes ${ }^{9-14}$ and electrons. ${ }^{13,14}$ High performance $p$ - and $n$ - type FETs were fabricated by employing $\mathrm{Pd},{ }^{11,14} \mathrm{In}^{15}$ or $\mathrm{Ag}^{15,16}$ electrodes, which are characterized by an optimal work function to match either the conduction (In and Ag) or the valence (Pd) band of $\mathrm{WSe}_{2}$. The choice of optimal metals ensures nearly-ohmic injection for one type of carrier (holes when Pd is used, electrons when In and Ag are used), but poor injection for the other carrier type, leading to unipolar transport. ${ }^{11,17}$

In order to observe ambipolar transport in $\mathrm{WSe}_{2}$, ionic liquid gating ${ }^{18-20}$ and ferroelectric polymers ${ }^{14}$ were used in order generate electric fields strong enough to align the valence and conduction band of $\mathrm{WSe}_{2}$ to the Fermi level of the electrodes. Using solid-state dielectric, ambipolar transport could only be observed by contacting the same $\mathrm{WSe}_{2}$ flake with a pair of electrodes composed of different metals, one ideal for electron- and the other one for hole-injection. ${ }^{17}$

Apart from metal contact engineering, other sophisticated device optimization strategies were adopted to achieve the highest unipolar mobilities reported for single layer $\mathrm{WSe}_{2}\left(\mu_{\mathrm{h}}>200 \mathrm{~cm}^{2} \mathrm{~V}^{-1} \mathrm{~s}^{-1}, \mu_{\mathrm{e}}>250\right.$ $\left.\mathrm{cm}^{2} \mathrm{~V}^{-1} \mathrm{~s}^{-1}\right)^{11,15}$, including encapsulation ${ }^{11,15}$, chemical doping ${ }^{11}$ and/or the use of high-k dielectrics ${ }^{15,20}$. On the contrary, very poor charge carrier mobility $\left(\mu_{\mathrm{h}}, \mu_{\mathrm{e}}<10^{-2} \mathrm{~cm}^{2} \mathrm{~V}^{-1} \mathrm{~s}^{-1}\right)$ and ION $/$ Ioff ratios $\left(<10^{2}\right)$ were achieved in single-layer WSe 2 FETs fabricated when using Au contacts and $\mathrm{SiO}_{2}$ substrates, ${ }^{21,22}$ which are 
the prototypical materials employed as electrodes and dielectrics in $2 \mathrm{D}$ electronics. For this reason, $\mathrm{WSe}_{2}$ remains less investigated than unipolar $\mathrm{MoS}_{2}$ in field-effect devices, despite its higher intrinsic mobility and ambipolarity.

In order to improve the device performances, the engineering of the energy levels of the 2DM through functionalization with molecules represents a valuable alternative to the use of diverse metals. ${ }^{9,23-29}$ In this context, self-assembled monolayers (SAMs), i.e. ultra-thin molecular layers that are covalently attached to the target surfaces, ${ }^{30}$ are particularly appealing since their ordered structure, framed by the self-assembly, ensures a parallel out-of-plane alignment of molecular dipoles, causing a profound change of surface energy and electronic properties of the functionalized surface. ${ }^{31,32}$ Structural defects in TMDs, such as vacancies and substitutional atoms, ${ }^{33,34}$ represent an ideal anchoring site for covalent functionalization. ${ }^{35,36}$ However, since defects are relatively rare in pristine TMDs, ${ }^{37}$ their functionalization leads to the presence of isolated molecules, ${ }^{37}$ and not to the formation of ordered SAMs that could be exploited to tune deterministically the energy levels of TMDs.

Here, we exploit functionalization with ordered molecular monolayers to demonstrate that Au electrodes can be used to inject efficiently both electrons and holes into single- and bi-layer $\mathrm{WSe}_{2}$ on $\mathrm{SiO}_{2}$. In particular, we demonstrate high performance unipolar $p$ - and $n$ - type transistors based on $\mathrm{WSe}_{2}$ single layers with top $\mathrm{Au}$ electrodes on $\mathrm{SiO}_{2}$, reaching mobilities as high as $\mu_{\mathrm{h}}=150 \mathrm{~cm}^{2} \mathrm{~V}^{-1} \mathrm{~s}^{-1}$ and $\mu_{\mathrm{e}}=17.9$ $\mathrm{cm}^{2} \mathrm{~V}^{-1} \mathrm{~s}^{-1}$ and IoN/IOFF ratio exceeding $10^{7}$ for the $p$-type transistors. Additionally, we provide opposite electronic functionalities by engineering a double-sided molecular functionalization to the two sides of bi-layer $\mathrm{WSe}_{2}$ flakes, thereby attaining almost balanced ambipolar transport with the same electrode metal on a solid-state dielectric. Such control over charge transport is achieved through functionalization of both $\mathrm{SiO}_{2}$ and $\mathrm{WSe}_{2}$ surfaces with molecules containing silane groups, which form ultra-thin, ordered and densely packed layers both on insulating $\mathrm{SiO}_{2}$ and on semiconducting $\mathrm{WSe}_{2}$. While the formation of such ordered layers on $\mathrm{SiO}_{2}$ is well established, our results indicate that silane groups covalently bind at defect 
sites naturally present at $\mathrm{WSe}_{2}$ surface, enabling the effective defect functionalization. On the same time, strong intermolecular interactions ensure the formation of a highly ordered molecular layer, which covers $\mathrm{WSe}_{2}$ surface evenly, making it possible to modify its electronic properties. In this way, we simultaneously achieve defect functionalization and energy level tuning, and ultimately engineer the charge transport in single and bi-layer $\mathrm{WSe}_{2}$.

\section{RESULTS AND DISCUSSION}

Trichloro(1H,1H,2H,2H-perfluorooctyl)silane

(PFS)

and

N-[3-

(Trimethoxysilyl)propyl]ethylenediamine (AHAPS) (see chemical structures in Fig. 1a and b) were selected for the functionalization of both $\mathrm{SiO}_{2}$ and $\mathrm{WSe}_{2}$, since the fluorine-rich PFS was demonstrated to induce $p$-doping effect on 2D materials, ${ }^{38}$ while the amine groups in AHAPS are expected to generate $n$-type doping. ${ }^{39}$ In order to engineer the electrical characteristics of $\mathrm{WSe}_{2}$, we followed two distinct approaches. First, mechanically exfoliated $\mathrm{WSe}_{2}$ single layers were transferred onto SAM-functionalized $\mathrm{SiO}_{2}$ substrates and subsequently contacted with metal electrodes (see Fig. 1c and d); second, devices based on $\mathrm{WSe}_{2}$ single layer on untreated $\mathrm{SiO}_{2}$ were exposed to vapors of two different molecules, deposited from vapor phase on top of the $\mathrm{WSe}_{2}$ single layer (see Fig. 1e and f).

Figures $1 \mathrm{c}$ and $\mathrm{d}$ show the device architecture and transfer characteristics of FETs based on a WSe 2 single layer on PFS- and AHAPS- treated $\mathrm{SiO}_{2}$ substrate, respectively. The formation of SAMs of both molecules on the $\mathrm{SiO}_{2}$ surface was confirmed through atomic force microscopy (AFM), X-ray photoelectron spectroscopy (XPS) and contact angle measurements (Fig. S1-S3). The transfer characteristics are also shown for a $\mathrm{WSe}_{2}$ single layer on untreated $\mathrm{SiO}_{2}$, which serves as a reference. Such reference device featured poor electrical properties (dashed line in Fig.1c and d), with very low drain source current ( $\mathrm{I}<1 \mathrm{nA}$ in the entire gate-voltage range explored in this study), corresponding to an electron mobility on the order of $10^{-6} \mathrm{~cm}^{2} \mathrm{~V}^{-1} \mathrm{~s}^{-1}$. Such poor performances, which are comparable to those reported for single-layer $\mathrm{WSe}_{2}$ on $\mathrm{SiO}_{2}$ substrates with $\mathrm{Au}$ electrodes, ${ }^{21,22}$ are caused by the almost- 
blocked charge injection from $\mathrm{Au}$ into $\mathrm{WSe}_{2}$, which introduces ultra-high contact resistance. When exfoliated and placed on top of the PFS SAM, WSe 2 based transistors displayed improved charge transport, with higher current balanced ambipolarity but still relatively low hole and electron mobilities $\left(\mu_{\mathrm{h}} \sim \mu_{\mathrm{e}} \sim 0.5 \mathrm{~cm}^{2} \mathrm{~V}^{-1} \mathrm{~s}^{-1}\right)$. Similarly, an AHAPS SAM induced strong $n$-doping, displaying an electron mobility approaching $0.4 \mathrm{~cm}^{2} \mathrm{~V}^{-1} \mathrm{~s}^{-1}$.

A different situation was encountered when exposing $\mathrm{WSe}_{2}$ single layer devices supported on untreated $\mathrm{SiO}_{2}$ substrates to the vapors of the two molecules. In this case, the devices were measured before and after top functionalization, so that the effect of the molecules on the electrical characteristics was directly addressed in the same device. Very poor electrical characteristics were measured for the as-fabricated devices (i.e. not exposed to PFS vapors), which are similar to the reference FET in Fig. 1a and 1b (dashed line in Fig. 1c and d). After exposure to PFS vapors, a five-orders-of-magnitude increase in the electrical current is observed, with the device exhibiting $p$-type transport with hole mobility $\mu_{\mathrm{h}}=20 \mathrm{~cm}^{2} \mathrm{~V}^{-1} \mathrm{~s}^{-1}$ (Fig. 1e). Similarly, exposure to AHAPS vapor causes a three-order-of-magnitude increase in the electrical current, with the device displaying an almost degenerated $n$-doping, with an $n$-type mobility of $7 \mathrm{~cm}^{2} \mathrm{~V}^{-}$ ${ }^{1} \mathrm{~S}^{-1}$ (Fig. 1f). An analogous performance improvement can be observed in the $\mathrm{WSe}_{2}$ output characteristics (Fig. S4). Before functionalization, the $\mathrm{I}_{\mathrm{DS}}-\mathrm{V}_{\mathrm{DS}}$ traces display very low current and are highly non-linear, indicating that a significant Schottky-like energy barrier develops at the interface between Au and untreated $\mathrm{WSe}_{2}$ (Fig. S4a). On the contrary, after functionalization, the $\mathrm{I}_{\mathrm{DS}}-\mathrm{V}_{\mathrm{DS}}$ traces become almost ideal (Fig. S4b).

We highlight here that PFS and AHAPS not only possess different functional groups, but also different anchoring groups, respectively trichlorosilane and trimethoxysilane. In order to verify whether different anchoring groups contribute to the effects observed on PFS- and AHAPS-treated WSe, we exposed $\mathrm{WSe}_{2}$ single layers to vapors of hydrochloric acid and methanol, which contain a - $\mathrm{Cl}$ atom and a -OMe group, in analogy to trichlorosilane and trimethoxysilane. Figure S5 shows that the electrical characteristics of 
$\mathrm{WSe}_{2}$ single-layer FETs are not modified significantly by exposure to either vapors, ruling out an important contribution of the anchoring groups to the modification of the $\mathrm{WSe}_{2}$ electrical characteristics shown in Fig. 1. Therefore, the different effect of PFS and AHAPS can be ascribed to the different functional groups, i.e. the fluorinated chains in PFS and the amine groups in AHAPS.

The treatment with PFS and AHAPS vapors also changes drastically the optical properties of $\mathrm{WSe}_{2}$. In particular, we characterized the photoluminescence (PL) of $\mathrm{WSe}_{2}$ single layers before and after the treatment with AHAPS and PFS at $77 \mathrm{~K}$, as shown in Fig. 1g,h. The pristine $\mathrm{WSe}_{2}$ single layer shows the typical spectral features, characterized by an exciton peak $\mathrm{X}_{0}$ at $1.73 \mathrm{eV}$ and a smaller peak at lower energy $(1.69 \mathrm{eV})$ corresponding to the negative trion $\mathrm{X}^{-} \cdot{ }^{40}$ A small peak is also visible at $1.60 \mathrm{eV}$, which was described as a defect-related exciton, indicating the presence of defects in the untreated $\mathrm{WSe}_{2}$ single layer. ${ }^{41}$ After exposure to PFS vapor, the intensity of the PL spectrum of the same flake was decreased by a factor 2, and its spectral features were significantly modified. In particular, the peak corresponding to the negative trion $\mathrm{X}^{-}$disappeared, while a additional feature arose at $1.71 \mathrm{eV}$, which can be explained as a positive trion peak $\mathrm{X}^{+}$(Fig. 1g). Since positive trions are bound states consisting of two holes and one electron, ${ }^{41}$ the increase in their relative abundance with respect to neutrally charged excitons is indicative of hole doping, in good agreement with the electrical characterization. In the case of the AHAPS treatment, the PL change after functionalization is even more important. After functionalization, the PL intensity decreases almost by one order of magnitude, and the most intense peak in the spectrum is the negative trion $\mathrm{X}^{-}$at $1.67 \mathrm{eV}$ (Fig. 1h), which indicates that the AHAPS introduces a high density of electrons. $^{40}$

While the effect of the functionalization of $\mathrm{SiO}_{2}$ on the electrical characteristics of $\mathrm{WSe}_{2}$ (architecture reported in Fig. 1c and d) could be expected on the basis of similar results reported for $\mathrm{MoS}_{2}{ }^{39}$, the improved charge injection and the consequent increase in mobility observed upon exposure of the $\mathrm{WSe}_{2}$ surface to vapor of different molecules (architecture reported in Fig. 1e and f) was unexpected. AFM 
imaging performed on PFS functionalized $\mathrm{WSe}_{2}$ reveals that the adlayer displays a rather flat morphology forming a discontinuous ultra-thin film with a surface coverage estimated to be less than 60\% (Fig. 2a). As evidenced in the topographical profile displayed in Fig. $2 \mathrm{~b}$ the height of the adlayer is $(1.2 \pm 0.4) \mathrm{nm}$, consistently with the $1.34 \mathrm{~nm}$ contour length of PFS, suggesting that the molecules are perpendicularly oriented on the surface of $\mathrm{WSe}_{2} .{ }^{42}$ In order to increase uniformity and surface coverage of the molecular layer, we treated $\mathrm{WSe}_{2}$ surface with ozone before exposing it to the molecular vapors (see Methods section). For ozone-treated $\mathrm{WSe}_{2}$, the morphology of the PFS layer is markedly different, as it covers completely the $\mathrm{WSe}_{2}$ surface (Fig. 2c). In the same way, a continuous thin film is obtained when an ozonetreated $\mathrm{WSe}_{2}$ layer is exposed to AHAPS vapors (Fig. 2d). Water contact angle measurements of pristine, PFS-treated and AHAPS-treated $\mathrm{WSe}_{2}$ surface are shown in Figs. S6. Interestingly, the water contact angles measured for the functionalized $\mathrm{WSe}_{2}$ surfaces are very similar to those measured for the functionalized $\mathrm{SiO}_{2}$ surfaces (Fig. S3), suggesting that the molecules are ordered in the same way in both cases.

Complementary, information on the molecular orientation was obtained by angle-resolved XPS. In particular, we observed that the core levels of the molecular adlayer and those of $\mathrm{WSe}_{2}$ are attenuated differently as a function of the angle (i.e. $0 \mathrm{deg}$ or $60 \mathrm{deg}$ ), indicating a preferential vertical orientation of the molecules with respect to the substrate normal (Fig. S7). All together, these data reveal that PFS and AHAPS form ordered monolayers not only on $\mathrm{SiO}_{2}$, but also on $\mathrm{WSe}_{2}$, and that the molecular ordering is analogous on the two surfaces.

Nevertheless, the interaction between $\mathrm{WSe}_{2}$ and the molecular layer is very different in case $\mathrm{WSe}_{2}$ single layers were transferred onto $\mathrm{SAM}$-functionalized $\mathrm{SiO}_{2}$ substrates (Fig. 1c,d) or treated with molecular vapors (Fig. 1e,f). In the first case, the bottom surface of $\mathrm{WSe}_{2}$ single layers is in contact with the exposed $\mathrm{F}$ or $\mathrm{NH}_{2}$ functional groups. On the contrary, in the second case AHAPS and PFS form an ordered molecular layer on top surface of the $\mathrm{WSe}_{2}$ in which the silanol groups lie close to the surface of $\mathrm{WSe}_{2}$, 
leading to completely different behaviors and resulting in different effects on the $\mathrm{WSe}_{2}$ electronic properties.

In order to understand the origin of the improved performances after molecular functionalization discussed in Fig.1, we performed ultraviolet photoemission spectroscopy (UPS) and XPS. In particular, while UPS provides valuable information on the work function changes in the system, XPS accesses the atomic core levels, allowing to describe the effect of molecular functionalization with elemental sensitivity. Figures $3 \mathrm{a}$ and $\mathrm{b}$ show the variation in the work function of pristine and functionalized $\mathrm{WSe}_{2}$ bulk crystals, as extracted by UPS. The presence of the AHAPS monolayer reduces the work function by $0.4 \mathrm{eV}$ indicating an $n$-type doping. In the case of the PFS monolayer, the functionalization results in a work function increase of $0.45 \mathrm{eV}$, which indicates $p$-type doping, in good agreement with the electrical characterization shown in Fig. 1e. We highlight that the shift in $\mathrm{WSe}_{2}$ energy levels also implies that charge carrier injection from $\mathrm{Au}$ is different. In particular, a significant Schottky energy barrier takes place at the interface between $\mathrm{Au}$ and untreated WSe2, impeding an efficient injection of electrons and holes. In these conditions, the device resistance is dominated by the high contact resistance, leading to the poor electrical performances shown in Fig, 1. On the contrary the increased (decreased) work function of PFS (AHAPS)-functionalized $\mathrm{WSe}_{2}$ results in the improved alignment between the $\mathrm{WSe}_{2}$ valence (conduction) band and $\mathrm{Au}$ work function, therefore favoring the injection of holes (electrons). In turn, the improved charge injection lowers the contact resistance, and permits to access the intrinsic electrical properties of $\mathrm{WSe}_{2}$, enabling the several-orders-of-magnitude improvement in the hole and electron mobility for treated $\mathrm{WSe}_{2}$.

The work function variation observed in this study is comparable to that measured for ordered SAMs of similar molecules, ${ }^{43,44}$ further confirming that the molecular monolayers on $\mathrm{WSe}_{2}$ are highly ordered and densely packed, leading to an excellent alignment of the molecular dipoles at the surface, which induces a local change in $\mathrm{WSe}_{2}$ energy levels. 
Figure $3 \mathrm{c}$ and $\mathrm{d}$ shows the XPS spectra of $\mathrm{W} 4 \mathrm{f}$ and Se $3 \mathrm{~d}$ core levels before and after AHAPS and PFS functionalization, respectively. The $\mathrm{W} 4 \mathrm{f}$ and Se $3 \mathrm{~d}$ peaks shift to higher (lower) binding energy after functionalization with AHAPS (PFS). In this regard, the shift in the position of the W $4 \mathrm{f}$ and Se $3 \mathrm{~d}$ core levels is in agreement with the change of the work function, i.e. a higher (lower) binding energy shift is observed when the work function is decreased (increased). This finding indicates that the energy levels of the uppermost $\mathrm{WSe}_{2}$ layers are affected by the presence of the molecular dipoles, which generate an electric field effect analogous to that of a gate electrode. ${ }^{43-45}$ Moreover, XPS also shows that the line shape of both W $4 \mathrm{f}$ and Se $3 \mathrm{~d}$ core levels remains almost unchanged upon functionalization with either molecules, except for the shift in energy. This finding indicates that the W-Se chemical bonds are not altered significantly, permitting to achieve excellent electrical performance in FETs WSe 2 .

In order to cast light onto the role of the silanol group, we measured the effect of other molecules containing a similar functional core yet exposing different anchoring groups on the electrical characteristics of $\mathrm{WSe}_{2}$ single layers. In particular, we focused our attention on PFS, and chose another two perfluorinated molecules with the same length but with different anchoring end groups, namely 2,2,3,3,4,4,5,5,6,6,7,7,8,8,8-Pentadecafluoro-1-octanol (PDFO) and 1H,1H,2H-Perfluoro-1-decene (PFD). While PFS possesses a trichlorosilane group that can be hydrolyzed into a silanol group, PDFO is terminated by an alcohol group, and PFD exhibits an alkene head. Figure 4 a-c shows the effect of the different perfluorinated molecules onto $\mathrm{WSe}_{2}$ single layers. As discussed above, the exposure of $\mathrm{WSe}_{2}$ single layers to PFS vapor introduces significant $p$-doping and an important increase in mobility (Fig. 4a). Instead, the exposure to PDFO vapor only results in a minor $p$-doping, with minimal effect on the mobility, which remains in the range $10^{-5}-10^{-6} \mathrm{~cm}^{2} \mathrm{~V}^{-1} \mathrm{~s}^{-1}$ (Fig. 4b). In the same way, an analogous treatment with PFD leads to a further degradation of the $\mathrm{WSe}_{2}$ electrical performances (Fig. 4c).

To elucidate the nature of the interaction between the top molecular monolayer and the $\mathrm{WSe}_{2}$ surface, we used XPS to characterize the surfaces of bulk $\mathrm{WSe}_{2}$ crystals exposed to vapors of the three different 
molecules and subsequently washed by sonication in ethanol and toluene. In particular, in Fig. 4d-f we show the F 1s core level spectra measured for PFS-, PDFO- and PDF-treated WSe, respectively. For the PFS-treated $\mathrm{WSe}_{2}$ (Fig. 4d), an intense F 1s peak is found, i.e. a significant amount of molecules still bound to the surface after sonication, indicating a strong interaction between PFS and WSe 2 . On the contrary, for the PDFO-treated surface (Fig. 4e), only traces of F atoms are measured after the washing step, demonstrating that the vast majority of molecules has been wash out. A similar situation is encountered for PFD (Fig. 4f), for which no F peak is observed after sonication.

Overall, the presence of PFS molecules on $\mathrm{WSe}_{2}$ after washing and sonication treatment suggests the formation of a stable interaction, which could be covalent in nature. However, the negligible change of W and Se core levels in the XPS spectra before and after molecular functionalization, rule out a massive covalent interaction. This apparent inconsistency could be explain considering that only a few molecules in the top monolayer form covalent bonds to isolated intrinsic defects in the $\mathrm{WSe}_{2}$ crystal structure. Indeed, the trichlorosilane group in PFS and the trimethoxysilane group in AHAPS can be hydrolyzed into a silanol group in presence of residual traces of water. ${ }^{46}$ In turn, the silanol groups can generate covalent bonds to oxygen substitutional atoms present in the $\mathrm{WSe}_{2}$ structure, ${ }^{47}$ and/or to Se vacancies, which were reported to form covalent bonds with $\mathrm{O} .{ }^{35}$ Additionally, it is well known that polymerization often takes place in silane-based SAMs, with bridges forming between silanol groups belonging to neighboring molecules. ${ }^{38}$ In this way, the PFS monolayer is composed of a polymerized layer of orderly aligned molecules, strongly anchored to the $\mathrm{WSe}_{2}$ surface through covalent bonds at sparse defect sites (Fig. 4g).

In contrast, the alcohol group of PDFO, which might bind to defects in $\mathrm{WSe}_{2}$, does not possess additional binding sites for polymerization; hence, most molecules are simply physisorbed on the $\mathrm{WSe}_{2}$ surface and are removed by the sonication procedure, which only leaves the few molecules chemisorbed 
at defect sites (Fig. 4h). Similarly, the alkene head of PFD prevents any covalent interaction with $\mathrm{WSe}_{2}$ (Fig. 4i), and the molecules are efficiently desorbed by sonication in solvents.

Our understanding of the anchoring mechanism for the PFS monolayer allows us to further engineer the functionalization. In particular, as discussed above, we found that an ozone treatment prior to the functionalization improves the coverage of the top monolayers. Indeed, the ozone treatment increases the number of $\mathrm{O}$ substitutional atoms in the structure of $\mathrm{WSe}_{2}$ single layers ${ }^{48}$ (as evidenced by XPS, see Fig. S8), which serve as anchoring sites for silanol groups, and thus for PFS and AHAPS attachment. Consequently, a higher number of PFS or AHAPS molecules will be able to be anchored to $\mathrm{WSe}_{2}$ to substitutional oxygen atoms introduced by the UV/Ozone treatment. This results in an improvement of the uniformity of the molecular layer. Therefore, we tested whether the improved coverage corresponds to a further improvement in the electrical performances of $\mathrm{WSe}_{2}$ field effect transistors. We treated three $\mathrm{WSe}_{2}$ field effect devices with a short ozone treatment (3 min) and subsequently with the PFS vapor. The short ozone treatment was found to introduce minor $p$-type doping in the electrical characteristics of $\mathrm{WSe}_{2}$, with only a slight increase in mobility, which remained below $10^{-1} \mathrm{~cm}^{2} \mathrm{~V}^{-1} \mathrm{~s}^{-1}$ (Fig. S9). After exposure to PFS vapor, all devices displayed stronger $p$-type doping and increased hole mobility as compared to the functionalization without ozone treatment (Fig. 5a). Interestingly, hole mobilities up to $150 \mathrm{~cm}^{2} \mathrm{~V}^{-1} \mathrm{~s}^{-1}$ were measured for ozone-exposed, PFS-treated $\mathrm{WSe}_{2}$ single layers contacted by Au electrodes. Moreover, in this case the doping does not degenerate, and an OFF current close to the detection limit IofF $=1 \mathrm{pA}$ can be reached, yielding an ION/IOFF ratio above $10^{7}$. Higher doping levels and improved mobilities were also observed for ozone-exposed, AHAPS-treated $\mathrm{WSe}_{2}$. In this case, electron mobilities up to $17.9 \mathrm{~cm}^{2} \mathrm{~V}^{-1} \mathrm{~s}^{-1}$ were measured in $\mathrm{WSe}_{2}$ single layers contacted by Au electrodes (Fig. 5b). Moreover, we found that the SAM functionalization provides protection against air exposure and temperature cycling (Fig. S10). In particular, after an initial degradation which lasted for 4 days, the electrical characteristics of PFS-based devices remained constant, displaying mobility $\mu_{\mathrm{h}}>10 \mathrm{~cm}^{2} \mathrm{~V}^{-1} \mathrm{~s}^{-1}$, and $\mathrm{I}_{\mathrm{ON}} / \mathrm{I}_{\mathrm{OFF}}>10^{5}$. 
Finally, we demonstrate that an asymmetric functionalization of the two surfaces of the same $\mathrm{WSe}_{2}$ bi- $^{-}$ layer flake (see architecture reported in Fig. 5c) offers the opportunity to harness ambipolar transport at the bi-layer limit. Towards this end, we fabricated a device based on a $\mathrm{WSe}_{2}$ bi-layer adsorbed on an AHAPS-functionalized $\mathrm{SiO}_{2}$ substrate, to induce $n$-type doping in the bottom layer. Subsequently, we treated the same bi-layer with PFS vapor, to induce $p$-type doping in the top layer. Figure 5c shows that ambipolar characteristics were measured in the as-obtained asymmetrically functionalized device, with almost balanced mobility $\left(\mu_{\mathrm{h}}=20 \mathrm{~cm}^{2} \mathrm{~V}^{-1} \mathrm{~s}^{-1}\right.$ and $\left.\mu_{\mathrm{e}}=5.7 \mathrm{~cm}^{2} \mathrm{~V}^{-1} \mathrm{~s}^{-1}\right)$, while retaining an ION/IOFF ratio exceeding $10^{3}$. On the contrary, for single layers $\mathrm{WSe}_{2}$, the same approach of asymmetric functionalization did not introduce ambipolar characteristics, and led to degraded charge transport (Fig. S11). This finding can be understood simply by considering that in the case of bi-layer $\mathrm{WSe}_{2}$ opposite polarity is provided to the top and bottom layers in contact with the different molecular monolayers, which contribute in parallel to the overall charge transport, giving rise to ambipolar transport. Instead, in the case of a single layer $\mathrm{WSe}_{2}$, the effects of the asymmetric functionalization cancel each other out, ultimately rendering an almost intrinsic $\mathrm{WSe}_{2}$, analogous to the pristine case.

\section{CONCLUSIONS}

In conclusion, we have demonstrated a chemical approach to control and improve charge transport in $\mathrm{WSe}_{2}$ single- and bi-layers. In particular, we have shown that silane-containing molecules form ordered monolayers on top of the $\mathrm{WSe}_{2}$ surface, which are similar to those formed by the same molecules on $\mathrm{SiO}_{2}$ in terms of molecular ordering and packing density. In contrast to previous works in which high performances $\mathrm{WSe}_{2}$ FETs were achieved by exploiting a more cumbersome method relying on the use as electrodes of metals with a work function matching the conduction or valence band of $\mathrm{WSe}_{2},{ }^{11,15}$ we demonstrate an alternative strategy by engineering the $\mathrm{WSe}_{2}$ energy levels to match the Au Fermi level. In this way, our molecularly engineered $\mathrm{WSe}_{2}$ single layers FET, contacted with conventional Au contacts on $\mathrm{SiO}_{2}$ dielectrics show $p$ - or $n$ - mobility as high as $\mu_{\mathrm{h}}=150 \mathrm{~cm}^{2} \mathrm{~V}^{-1} \mathrm{~s}^{-1}$ and $\mu_{\mathrm{e}}=17.9 \mathrm{~cm}^{2} \mathrm{~V}^{-1} \mathrm{~s}^{-1}$. 
Moreover, we demonstrated ambipolar transport in bi-layer WSe 2 contacted with the same electrode metal through a double asymmetric functionalization of its top and bottom surfaces. By combining energy level engineering and defect functionalization, silane-based monolayers offer the possibility to provide the desired charge transport characteristics to atomically thin $\mathrm{WSe}_{2}$ and hence demonstrate high-performance field-effect devices. Based on our results, we anticipate that silane-based monolayers can be employed to couple other molecular functions to Se-based transition metal dichalcogenides, including specific molecular capabilities with no analogue in $2 \mathrm{D}$ materials. ${ }^{49}$ Furthermore, local patterning of the two surfaces of the 2D semiconductor may allow bottom-up engineering of lateral heterojunctions.

\section{EXPERIMENTAL METHODS}

\section{Sample preparation}

$\mathrm{SiO}_{2} / \mathrm{Si}-\mathrm{n}^{++}$substrates (from Fraunhofer Institute for Photonic Microsystems IPMS, Dresden, Germany) with $90 \mathrm{~nm}$ thick oxide were cleaned in an ultrasonic bath in acetone and isopropanol and dried under nitrogen flow. Afterwards, the substrates were treated by $\mathrm{UV} / \mathrm{O}_{3}$ for $20 \mathrm{~min}$. For the growth of PFS (Sigma Aldrich) $\mathrm{SAM}$ on $\mathrm{SiO}_{2}$, the freshly $\mathrm{UV} / \mathrm{O}_{3}$ treated substrates were placed in a sealed reactor containing $20 \mu \mathrm{L}$ PFS, and heated at $75^{\circ} \mathrm{C}$ for $90 \mathrm{~min}$. Once cooled back to room temperature, the substrates were rinsed with $20 \mathrm{~mL}$ toluene, sonicated $10 \mathrm{~s}$ in ethanol, and baked on a hotplate at $60{ }^{\circ} \mathrm{C}$ for $1 \mathrm{~h}$. For the growth of AHAPS (Sigma Aldrich) SAM, the freshly $\mathrm{UV} / \mathrm{O}_{3}$-treated substrates were placed in $10 \mathrm{~mL}$ toluene containing $7 \mu \mathrm{L}$ AHAPS. The solution was then heated at $60{ }^{\circ} \mathrm{C}$ for $1 \mathrm{~h}$ and then left at room temperature for $11 \mathrm{~h}$. The substrates were then rinsed with $20 \mathrm{~mL}$ toluene, sonicated in hexane for $5 \mathrm{~s}$, and baked on a hotplate for $1 \mathrm{~h}$ at $60^{\circ} \mathrm{C}$. The AHAPS treated substrates were then immersed in $10 \mathrm{~mL}$ toluene with $50 \mu \mathrm{L}$ triethylamine for $5 \mathrm{~min}$, and dried on a hotplate at $60^{\circ} \mathrm{C}$ for $5 \mathrm{~min}$. The SAM-functionalized surfaces were characterized by water contact angle (Krüss DSA 100) measurements upon deposition of a 
$3-\mu \mathrm{L}$ drop of milliQ water on the surface. Atomically thin $\mathrm{WSe}_{2}$ flakes were exfoliated via a stamp method, using a silicone-based gel film (PF film, retention level X4, gel thickness 6.5 mil) from Gel-Pak on a synthetic $\mathrm{WSe}_{2}$ single crystal (purchased from $\mathrm{HQ}$ Graphene). The single-layer and bi-layer $\mathrm{WSe}_{2}$ flakes were identified by optical contrast (Olympus BX51) and PL spectroscopy (Renishaw microscope equipped with a $100 \mathrm{x}$ objective, $\lambda_{\mathrm{ex}}=532 \mathrm{~nm}$, spot size of $800 \mathrm{~nm}$ ). The low temperature PL characterization was performed with a Linkam THMS600 temperature-controlled stage with a LNP95 cooling system. Using the shadow mask approach (homemade shadow mask, Fig. S12) or the photolithographic approach (AZ1505 photoresist and AZ326 MIF developer from MicroChemicals, Laserwriter LW405B from Microtech), $30 \mathrm{~nm}$ gold were thermally evaporated with a Plassys MEB 300, forming the drain and source electrodes on $\mathrm{WSe}_{2}$. For the vapor treatment of $\mathrm{WSe}_{2}, 20 \mu \mathrm{L} \mathrm{PFS}$, AHAPS, PDFO (Sigma Aldrich) or PFD (Sigma Aldrich) were poured into a sealed reactor with the samples to treat, and the system was heated at $75{ }^{\circ} \mathrm{C}$ for $90 \mathrm{~min}$. When mentioned, a $3 \mathrm{~min} \mathrm{UV} / \mathrm{Ozone}$ treatment was performed with a Novascan PDS Pro Series. Table S1 summarizes the average mobility and ON-OFF ratio measured in $\mathrm{WSe}_{2}$-based FETs treated in the different ways.

\section{Sample characterization}

The devices were electrically characterized by a Keithley dual channel 2636 Sourcemeter, in a threeterminal configuration with the back gate contacted through an underlying metal plate, in a glovebox filled with nitrogen, in dark conditions.

\section{XPS/UPS analyses}

X-ray photoelectron spectroscopy (XPS) was carried out with a non-monochromatized $\mathrm{Mg} \mathrm{K}$ source (emission line at $1253.6 \mathrm{eV}$ ), and ultraviolet photoelectron spectroscopy (UPS) was performed by means of the He I photon at about $21.2 \mathrm{eV}$. The electron energy analyzer is a VSW HSA100 hemispherical analyzer with PSP electronic power supply and control, the total energy resolution is $0.8 \mathrm{eV}$ for XPS and about $0.1 \mathrm{eV}$ for UPS. The binding energy (BE) scale of XPS spectra was calibrated by using the Au $4 \mathrm{f}$ 
peak at $84.0 \mathrm{eV}$ as a reference. The secondary electron cutoff (SECO) spectra were measured with a sample bias of $-7.0 \mathrm{~V}$.

\section{AFM imaging}

AFM measurements were carried out using a Veeco Dimension 3100 atomic force microscope, under the control of a Nanoscope IV unit in ambient conditions. Topographic and phase imaging were recorded while operating in tapping mode, with an antimony $n$-doped silicon cantilever.

\section{ASSOCIATED CONTENT}

\section{Supporting Information}

The Supporting Information is available free of charge on the ACS Publications website at DOI: xxxxx.

Figures S1-S12, Table 1 and references (refs 1-5). Characterization of PFS and AHAPS SAMs on $\mathrm{SiO}_{2}$ surfaces; electrical performances of $\mathrm{WSe}_{2}$ single layers treated by vapors of $\mathrm{HCl}$ and methanol. Water contact angle of pristine, PFS-treated and AHAPS-treated WSe 2 surface. Angle Resolved XPS of AHAPS and PFS functionalized WSe 2 . Output characteristics of $\mathrm{WSe}_{2}$ FET devices before and after treatment. Effect of ozone treatment on the spectroscopic and electrical characteristics of $\mathrm{WSe}_{2}$. Air and temperature stability on the electrical characteristics of $\mathrm{WSe}_{2}$. Electrical performances of double functionalized $\mathrm{WSe}_{2}$ single. Summary of averaged mobilities and $\mathrm{I}_{\mathrm{ON}} / \mathrm{I}_{\mathrm{OFF}}$ of field effect transistors based on functionalized $\mathrm{WSe}_{2}$ single layers. (PDF)

The authors declare no competing financial interests.

\section{AUTHOR INFORMATION}

\section{Corresponding Authors}


Paolo Samorì samori@,unistra.fr; Marco Gobbi marco_gobbi001@ehu.eus; Emanuele Orgiu emanuele.orgiu@emt.inrs.ca

\section{Author Contributions}

M.-A.S., M.G., E.O. and P.S. conceived the experiment. M.-A.S. performed the spectroscopic characterization and carried out the device fabrication and characterization with the aid of T.L. and Y.W.. M.E. carried out the AFM study; S.B. optimized the sample preparation. R.V., M.T. and M.V.N. performed the XPS and UPS experiments. M.G. and M.-A.S. co-wrote the paper. All authors discussed the results and contributed to the interpretation of data as well as to editing the manuscript.

\section{ACKNOWLEDGMENT}

We acknowledge funding from the European Commission through the Graphene Flagship Core 2 project (GA-785219), the Marie Sklodowska-Curie IEF SUPER2D (GA-748971) and IEF - GALACTIC (GA2014-628563); the Agence Nationale de la Recherche through the Labex projects CSC (ANR-10-LABX0026 CSC) and NIE (ANR-11-LABX-0058 NIE) within the Investissement d'Avenir program (ANR-10120 IDEX-0002-02), the International Center for Frontier Research in Chemistry (icFRC) as well as the

Chinese Scholarship Council. E. O. is supported by the Natural Sciences and Engineering Research Council of Canada (NSERC), through an individual Discovery Grant, and by Fonds de recherche du Québec - Nature et Technologies (FRQNT).

\section{REFERENCES}

(1) Wang, Q. H.; Kalantar-Zadeh, K.; Kis, A.; Coleman, J. N.; Strano, M. S. Electronics and Optoelectronics of Two-Dimensional Transition Metal Dichalcogenides. Nat. Nanotechnol. 2012, 7, 699-712.

(2) Fiori, G.; Bonaccorso, F.; Iannaccone, G.; Palacios, T.; Neumaier, D.; Seabaugh, A.; Banerjee, S. K.; Colombo, L. Electronics Based on Two-Dimensional Materials. Nat. Nanotechnol. 2014, 9, $768-779$. 
(3) Novoselov, K. S. Electric Field Effect in Atomically Thin Carbon Films. Science 2004, 306, 666669.

(4) Chhowalla, M.; Shin, H. S.; Eda, G.; Li, L.-J.; Loh, K. P.; Zhang, H. The Chemistry of TwoDimensional Layered Transition Metal Dichalcogenide Nanosheets. Nat. Chem. 2013, 5, 263-275.

(5) Jariwala, D.; Sangwan, V. K.; Lauhon, L. J.; Marks, T. J.; Hersam, M. C. Emerging Device Applications for Semiconducting Two-Dimensional Transition Metal Dichalcogenides. ACS Nano 2014, 8, 1102-1120.

(6) Chhowalla, M.; Jena, D.; Zhang, H. Two-Dimensional Semiconductors for Transistors. Nat. Rev. Mater. 2016, 1, 16052.

(7) Li, S.-L.; Tsukagoshi, K.; Orgiu, E.; Samorì, P. Charge Transport and Mobility Engineering in Two-Dimensional Transition Metal Chalcogenide Semiconductors. Chem. Soc. Rev. 2016, 45, $118-151$.

(8) Yu, L.; Zubair, A.; Santos, E. J. G.; Zhang, X.; Lin, Y.; Zhang, Y.; Palacios, T. High-Performance WSe2 Complementary Metal Oxide Semiconductor Technology and Integrated Circuits. Nano Lett. 2015, 15, 4928-4934.

(9) Chen, C.-H.; Wu, C.-L.; Pu, J.; Chiu, M.-H.; Kumar, P.; Takenobu, T.; Li, L.-J. Hole Mobility Enhancement and $p$-Doping in Monolayer WSe2 by Gold Decoration. 2D Mater. 2014, 1, 034001.

(10) Movva, H. C. P.; Rai, A.; Kang, S.; Kim, K.; Fallahazad, B.; Taniguchi, T.; Watanabe, K.; Tutuc, E.; Banerjee, S. K. High-Mobility Holes in Dual-Gated WSe2 Field-Effect Transistors. ACS Nano 2015, 9, 10402-10410.

(11) Fang, H.; Chuang, S.; Chang, T. C.; Takei, K.; Takahashi, T.; Javey, A. High-Performance Single Layered WSe2 p-FETs with Chemically Doped Contacts. Nano Lett. 2012, 12, 3788-3792.

(12) Podzorov, V.; Gershenson, M. E.; Kloc, Ch.; Zeis, R.; Bucher, E. High-Mobility Field-Effect Transistors Based on Transition Metal Dichalcogenides. Appl. Phys. Lett. 2004, 84, 3301-3303.

(13) Zhou, H.; Wang, C.; Shaw, J. C.; Cheng, R.; Chen, Y.; Huang, X.; Liu, Y.; Weiss, N. O.; Lin, Z.; Huang, Y.; Duan, X. Large Area Growth and Electrical Properties of $p$-Type WSe2 Atomic Layers. Nano Lett. 2015, 15, 709-713.

(14) Li, D.; Wang, X.; Chen, Y.; Zhu, S.; Gong, F.; Wu, G.; Meng, C.; Liu, L.; Wang, L.; Tie Lin; Sun, S.; Shen, H.; Wang, X.; Hu, W.; Wang, J.; Sun, J.; Meng, X.; Chu, J. The Ambipolar Evolution of a High-Performance WSe2 Transistor Assisted by a Ferroelectric Polymer. Nanotechnology 2018, 29, 105202.

(15) Liu, W.; Kang, J.; Sarkar, D.; Khatami, Y.; Jena, D.; Banerjee, K. Role of Metal Contacts in Designing High-Performance Monolayer $n$-Type WSe2 Field Effect Transistors. Nano Lett. 2013, 13, 1983-1990.

(16) Li, S.; Wang, S.; Tang, D.-M.; Zhao, W.; Xu, H.; Chu, L.; Bando, Y.; Golberg, D.; Eda, G. HalideAssisted Atmospheric Pressure Growth of Large WSe2 and WS2 Monolayer Crystals. Appl. Mater. Today 2015, 1, 60-66.

(17) Das, S.; Appenzeller, J. WSe2 Field Effect Transistors with Enhanced Ambipolar Characteristics. Appl. Phys. Lett. 2013, 103, 103501.

(18) Chuang, H.-J.; Tan, X.; Ghimire, N. J.; Perera, M. M.; Chamlagain, B.; Cheng, M. M.-C.; Yan, J.; Mandrus, D.; Tománek, D.; Zhou, Z. High Mobility WSe2 $p$ - and $n$-Type Field-Effect Transistors Contacted by Highly Doped Graphene for Low-Resistance Contacts. Nano Lett. 2014, 14, 3594 3601 .

(19) Huang, J.-K.; Pu, J.; Hsu, C.-L.; Chiu, M.-H.; Juang, Z.-Y.; Chang, Y.-H.; Chang, W.-H.; Iwasa, Y.; Takenobu, T.; Li, L.-J. Large-Area Synthesis of Highly Crystalline WSe2 Monolayers and Device Applications. ACS Nano 2014, 8, 923-930.

(20) Zhang, Y. J.; Oka, T.; Suzuki, R.; Ye, J. T.; Iwasa, Y. Electrically Switchable Chiral Light-Emitting Transistor. Science 2014, 344, 725-728. 
(21) Neupane, G. P.; Tran, M. D.; Yun, S. J.; Kim, H.; Seo, C.; Lee, J.; Han, G. H.; Sood, A. K.; Kim, J. Simple Chemical Treatment to $n$-Dope Transition-Metal Dichalcogenides and Enhance the Optical and Electrical Characteristics. ACS Appl. Mater. \& Interfaces 2017, 9, 11950-11958.

(22) Pudasaini, P. R.; Oyedele, A.; Zhang, C.; Stanford, M. G.; Cross, N.; Wong, A. T.; Hoffman, A. N.; Xiao, K.; Duscher, G.; Mandrus, D. G.; Ward, T. Z.; Rack, P. D. High-Performance Multilayer WSe2 Field-Effect Transistors with Carrier Type Control. Nano Res 2018, 11, 722-730.

Gobbi, M.; Orgiu, E.; Samorì, P. When 2D Materials Meet Molecules: Opportunities and Challenges of Hybrid Organic/Inorganic van Der Waals Heterostructures. Adv. Mater. 2018, 30, 1706103.

Bertolazzi, S.; Gobbi, M.; Zhao, Y.; Backes, C.; Samorì, P. Molecular Chemistry Approaches for Tuning the Properties of Two-Dimensional Transition Metal Dichalcogenides. Chem. Soc. Rev. 2018, 47, 6845-6888.

(25) Ryder, C. R.; Wood, J. D.; Wells, S. A.; Hersam, M. C. Chemically Tailoring Semiconducting Two-Dimensional Transition Metal Dichalcogenides and Black Phosphorus. ACS Nano 2016, 10, 3900-3917.

(26) Kang, D.-H.; Shim, J.; Jang, S. K.; Jeon, J.; Jeon, M. H.; Yeom, G. Y.; Jung, W.-S.; Jang, Y. H.; Lee, S.; Park, J.-H. Controllable Nondegenerate $p$-Type Doping of Tungsten Diselenide by Octadecyltrichlorosilane. ACS Nano 2015, 9, 1099-1107.

(27) Mosciatti, T.; Haar, S.; Liscio, F.; Ciesielski, A.; Orgiu, E.; Samorì, P. A Multifunctional PolymerGraphene Thin-Film Transistor with Tunable Transport Regimes. ACS Nano 2015, 9, 2357-2367.

(28) Daukiya, L.; Seibel, J.; Feyter, S. D. Chemical Modification of 2D Materials Using Molecules and Assemblies of Molecules. Adv. Phys. 2019, 4, 1625723.

(29) Cheng, L.; Lee, J.; Zhu, H.; Ravichandran, A. V.; Wang, Q.; Lucero, A. T.; Kim, M. J.; Wallace, R. M.; Colombo, L.; Kim, J. Sub-10 Nm Tunable Hybrid Dielectric Engineering on MoS2 for TwoDimensional Material-Based Devices. ACS Nano 2017, 11, 10243-10252.

(30) DiBenedetto, S. A.; Facchetti, A.; Ratner, M. A.; Marks, T. J. Molecular Self-Assembled Monolayers and Multilayers for Organic and Unconventional Inorganic Thin-Film Transistor Applications. Adv. Mater. 2009, 21, 1407-1433.

(31) Lee, W. H.; Park, Y. D. Tuning Electrical Properties of 2D Materials by Self-Assembled Monolayers. Adv. Mater. Interfaces 2017, 5, 1700316.

(32) Seber, G.; Rudnev, A. V.; Droghetti, A.; Rungger, I.; Veciana, J.; Mas-Torrent, M.; Rovira, C.; Crivillers, N. Covalent Modification of Highly Ordered Pyrolytic Graphite with a Stable Organic Free Radical by Using Diazonium Chemistry. Chem.: Eur. J. 2017, 23, 1415-1421.

(33) Lin, Z.; Carvalho, B. R.; Kahn, E.; Lv, R.; Rao, R.; Terrones, H.; Pimenta, M. A.; Terrones, M. Defect Engineering of Two-Dimensional Transition Metal Dichalcogenides. 2D Mater. 2016, 3, 022002.

(34) Barja, S.; Refaely-Abramson, S.; Schuler, B.; Qiu, D. Y.; Pulkin, A.; Wickenburg, S.; Ryu, H.; Ugeda, M. M.; Kastl, C.; Chen, C.; Hwang, C.; Schwartzberg, A.; Aloni, S.; Mo, S.-K.; Ogletree, D. F.; Crommie, M. F.; Yazyev, O. L.; Louie, S. G.; Neaton, J. B.; Weber-Bargioni, A. Identifying Substitutional Oxygen as a Prolific Point Defect in Monolayer Transition Metal Dichalcogenides. Nat. Commun. 2019, 10, 3382

(35) Zhao, P.; Kiriya, D.; Azcatl, A.; Zhang, C.; Tosun, M.; Liu, Y.-S.; Hettick, M.; Kang, J. S.; McDonnell, S.; Kc, S.; Guo, J. Cho, K.; Wallace, R. M.; Javey, A. Air Stable $p$-Doping of WSe2 by Covalent Functionalization. ACS Nano 2014, 8, 10808-10814.

(36) Schmidt, H.; Giustiniano, F.; Eda, G. Electronic Transport Properties of Transition Metal Dichalcogenide Field-Effect Devices: Surface and Interface Effects. Chem. Soc. Rev. 2015, 44, 7715-7736. 
(37) Bertolazzi, S.; Bonacchi, S.; Nan, G.; Pershin, A.; Beljonne, D.; Samorì, P. Engineering Chemically Active Defects in Monolayer MoS2 Transistors via Ion-Beam Irradiation and Their Healing via Vapor Deposition of Alkanethiols. Adv. Mater. 2017, 29, 1606760.

(38) Lee, B.; Chen, Y.; Duerr, F.; Mastrogiovanni, D.; Garfunkel, E.; Andrei, E. Y.; Podzorov, V. Modification of Electronic Properties of Graphene with Self-Assembled Monolayers. Nano Lett. 2010, 10, 2427-2432.

(39) Li, Y.; Xu, C.-Y.; Hu, P.; Zhen, L. Carrier Control of MoS2 Nanoflakes by Functional SelfAssembled Monolayers. ACS Nano 2013, 7, 7795-7804.

(40) Jones, A. M.; Yu, H.; Ghimire, N. J.; Wu, S.; Aivazian, G.; Ross, J. S.; Zhao, B.; Yan, J.; Mandrus, D. G.; Xiao, D.; Yao, W.; Xu, X. Optical Generation of Excitonic Valley Coherence in Monolayer WSe2. Nat. Nanotechnol. 2013, 8, 634-638.

(41) Wu, Z.; Luo, Z.; Shen, Y.; Zhao, W.; Wang, W.; Nan, H.; Guo, X.; Sun, L.; Wang, X.; You, Y.; $\mathrm{Ni}, \mathrm{Z}$. Defects as a Factor Limiting Carrier Mobility in WSe2: A Spectroscopic Investigation. Nano Research 2016, 9, 3622-3631.

(42) Sugimura, H.; Hayashi, K.; Saito, N.; Nakagiri, N.; Takai, O. Surface Potential Microscopy for Organized Molecular Systems. Appl. Surf. Sci. 2002, 188, 403-410.

(43) Timpel, M.; Nardi, M. V.; Ligorio, G.; Wegner, B.; Pätzel, M.; Kobin, B.; Hecht, S.; Koch, N. Energy-Level Engineering at $\mathrm{ZnO}$ /Oligophenylene Interfaces with Phosphonate-Based SelfAssembled Monolayers. ACS Appl. Mater. Interfaces 2015, 7, 11900-11907.

(44) Timpel, M.; Nardi, M. V.; Krause, S.; Ligorio, G.; Christodoulou, C.; Pasquali, L.; Giglia, A.; Frisch, J.; Wegner, B.; Moras, P.; Koch, N. Surface Modification of $\mathrm{ZnO}(0001)-Z n$ with Phosphonate-Based Self-Assembled Monolayers: Binding Modes, Orientation, and Work Function. Chem. Mater. 2014, 26, 5042-5050.

(45) Gobbi, M.; Bonacchi, S.; Lian, J. X.; Liu, Y.; Wang, X.-Y.; Stoeckel, M.-A.; Squillaci, M. A.; D’Avino, G.; Narita, A.; Müllen, K.; Feng, X.; Olivier, Y.; Beljonne, D.; Samorì, P.; Orgiu, E. Periodic Potentials in Hybrid van Der Waals Heterostructures Formed by Supramolecular Lattices on Graphene. Nat. Commun. 2017, 8, 14767.

(46) Ulman, A. Formation and Structure of Self-Assembled Monolayers. Chem. Rev. 1996, 96, 15331554.

(47) Ta, T. K. H.; Tran, T. N. H.; Tran, Q. M. N.; Pham, D. P.; Pham, K. N.; Cao, T. T.; Kim, Y. S.; Tran, D. L.; Ju, H.; Phan, B. T. Surface Functionalization of WO3 Thin Films with (3Aminopropyl)Triethoxysilane and Succinic Anhydride. J. Elec. Mater. 2017, 46, 3345-3352.

(48) Wang, S.; Zhao, W.; Giustiniano, F.; Eda, G. Effect of Oxygen and Ozone on $p$-Type Doping of Ultra-Thin WSe2 and MoSe2 Field Effect Transistors. Phys. Chem. Chem. Phys. 2016, 18, 43044309.

(49) Gobbi, M.; Bonacchi, S.; Lian, J. X.; Vercouter, A.; Bertolazzi, S.; Zyska, B.; Timpel, M.; Tatti, R.; Olivier, Y.; Hecht, S.; Nardi, M. V.; Beljonne, D.; Orgiu, E.; Samorì, P. Collective Molecular Switching in Hybrid Superlattices for Light-Modulated Two-Dimensional Electronics. Nat. Commun. 2018, 9, 2661. 

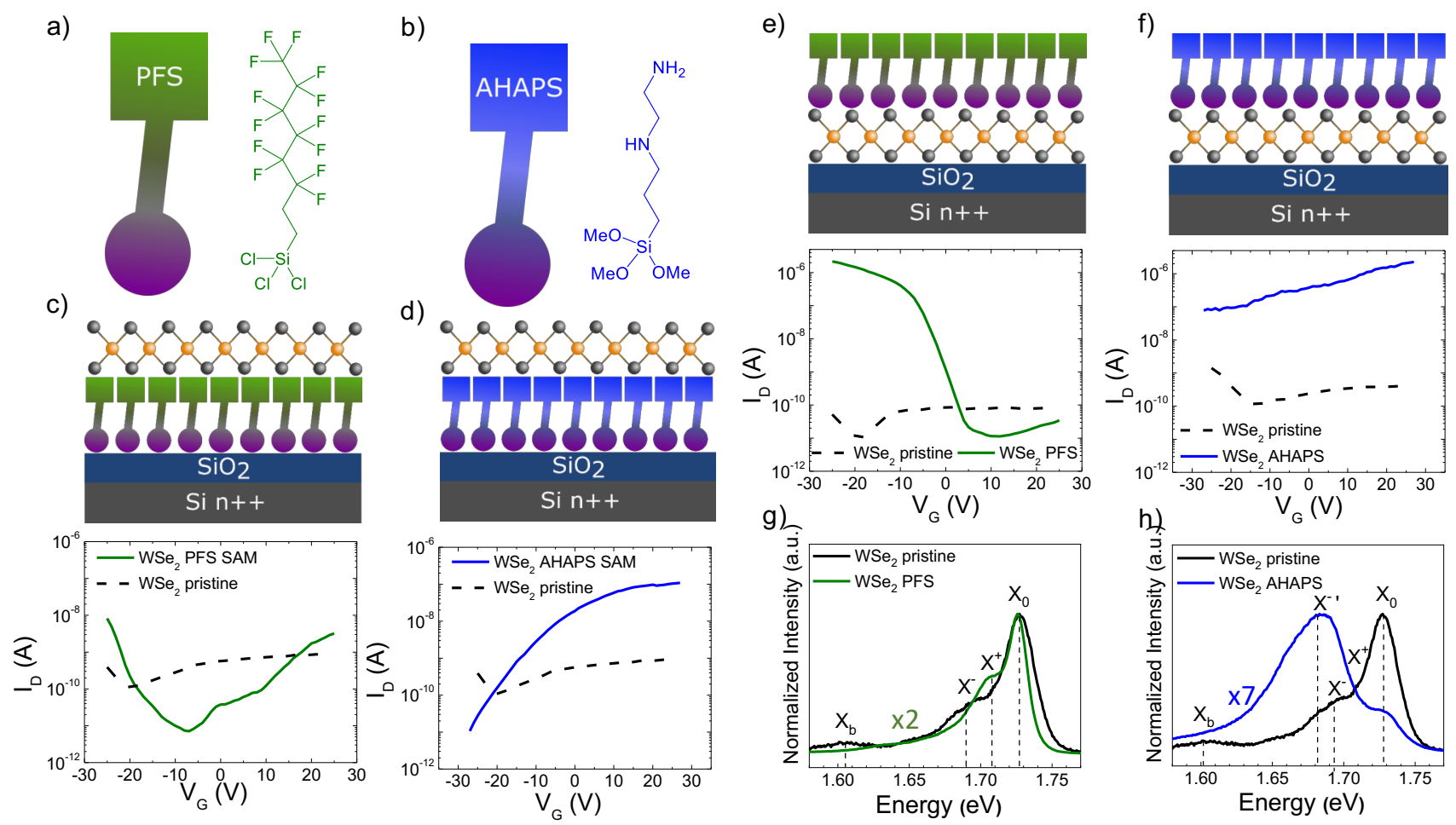

Figure 1. (a,b) Chemical structure of PFS and AHAPS. (c,d) Device architecture and corresponding transfer curve of single-layer $\mathrm{WSe}_{2}$ flakes measured with gold contacts on (c) PFS-treated and (d) AHAPS-treated $\mathrm{SiO}_{2}$ dielectric (90 nm thick) Channel length $\mathrm{L}$ and width $\mathrm{W}$ : (c) $\mathrm{L}=2.6 \mu \mathrm{m} ; \mathrm{W}=4 \mu \mathrm{m}$ (d) $\mathrm{L}=6 \mu \mathrm{m} ; \mathrm{W}=4.6 \mu \mathrm{m}$. For the sake of comparison, the transfer curve of a reference device based on a $\mathrm{WSe}_{2}$ single layer on untreated $\mathrm{SiO}_{2}$ surface is also shown (dashed black lines, $\mathrm{L}=5.4 \mu \mathrm{m} ; \mathrm{W}=2.2 \mu \mathrm{m}$ ). (e,f) Device architecture and corresponding transfer curve of $\mathrm{WSe}_{2}$ devices treated with e) PFS and f) AHAPS vapor on untreated $\mathrm{SiO}_{2}$ dielectric. The transfer curves of the same $\mathrm{WSe}_{2}$ devices before molecular treatment are shown as dashed black lines. (e) $\mathrm{L}=3.1 \mu \mathrm{m} ; \mathrm{W}=1.3 \mu \mathrm{m}$; (f) $\mathrm{L}=3.6 \mu \mathrm{m} ; \mathrm{W}=1.2$ $\mu \mathrm{m}$; (g,h) Photoluminescence spectra of $\mathrm{WSe}_{2}$ single layers at $77 \mathrm{~K}$ treated with (g) PFS and (h) AHAPS vapors. The photoluminescence spectra of the pristine $\mathrm{WSe}_{2}$ single layers are shown in black. 
a)
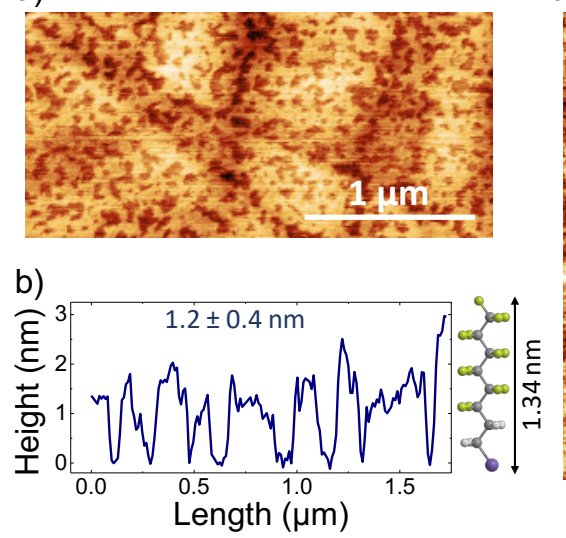

c)

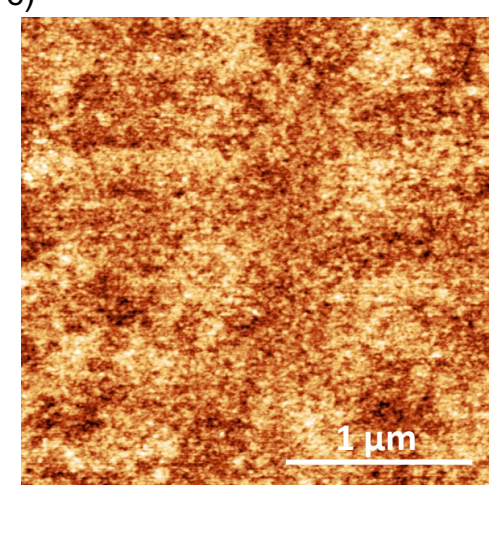

d)

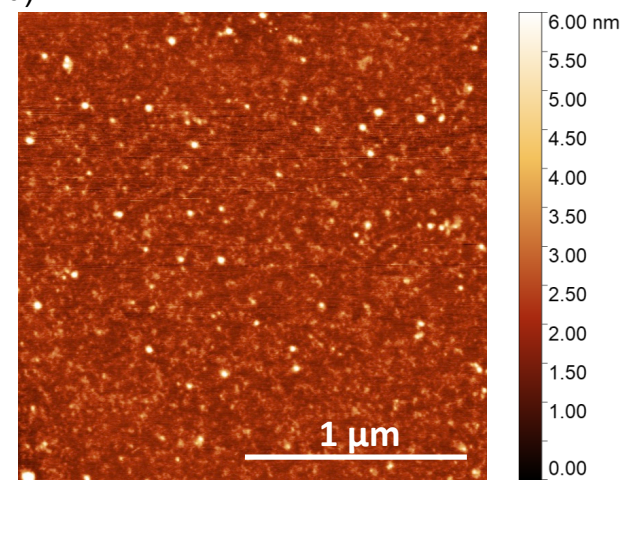

Figure 2. (a) AFM image of the surface of a $\mathrm{WSe}_{2}$ bulk crystal exposed to PFS vapor. A relatively flat layer covers approximately $60 \%$ of the surface. (b) Height profile extracted from image (a). (c,d) AFM images of the surface of a WSe 2 bulk crystal exposed to (c) PFS and (d) AHAPS vapor after ozone treatment. The vertical scale bar is the same. 
a)
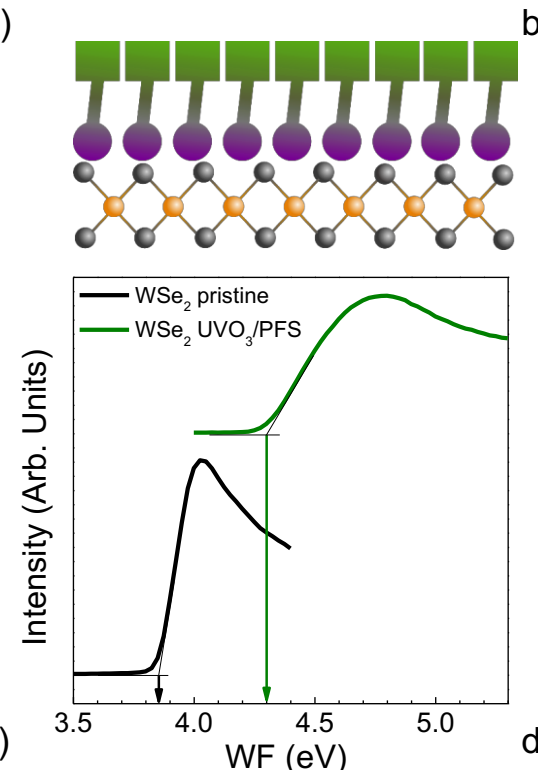

c)

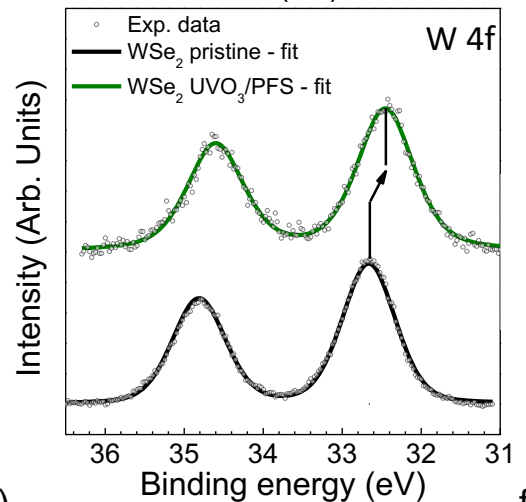

e)

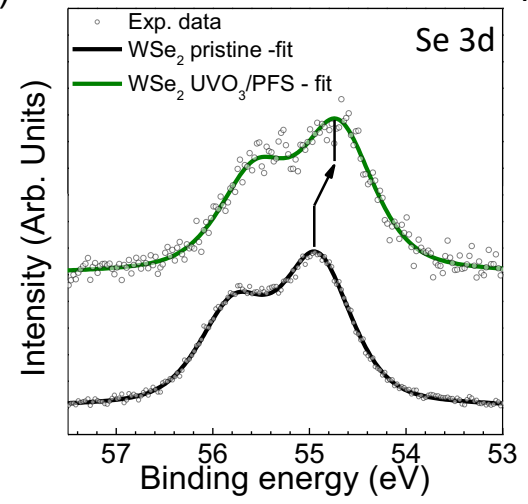

b)
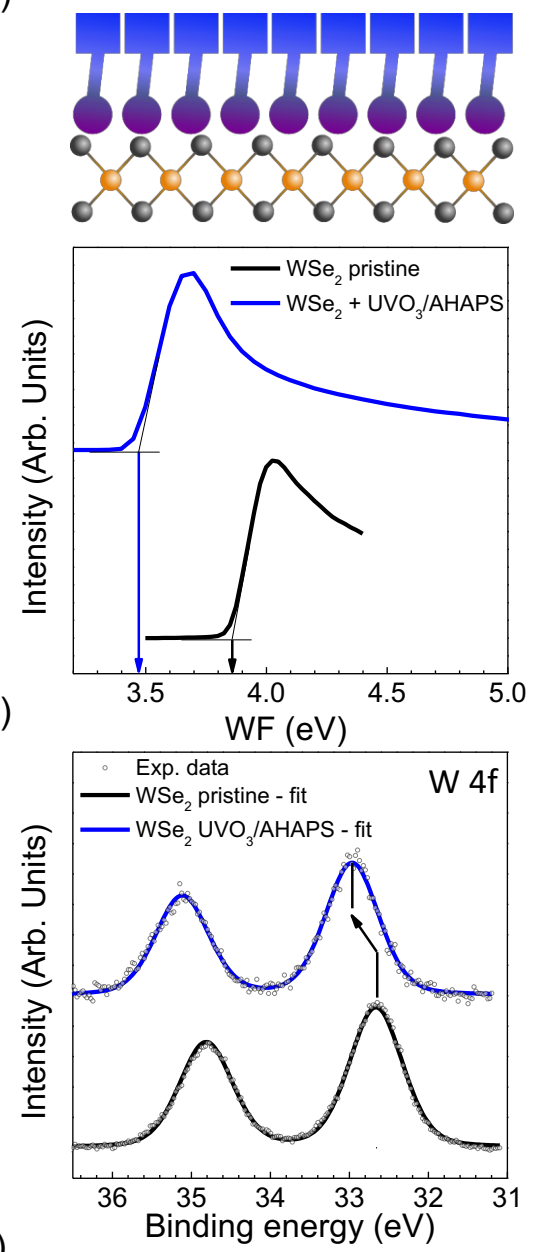

f)

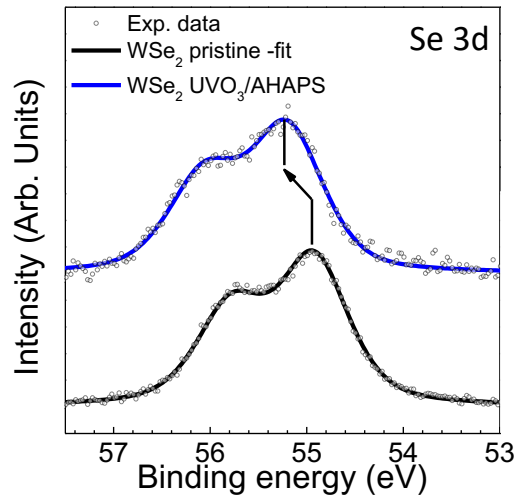

Figure 3. $(a, b)$ Secondary electron cutoff of (a) AHAPS-treated and (b) PFS-treated WSe $\mathrm{Wurface}_{2}$, compared to the corresponding pristine surface. (c,d) W 4 f core level spectra before and after (c) AHAPS and (d) PFS functionalization. (e,f) Se 3d core level spectra before and after (c) AHAPS and (d) PFS functionalization. 
a)

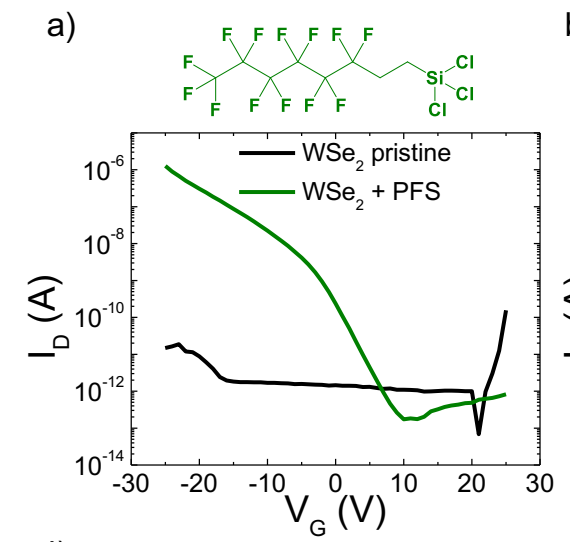

d)

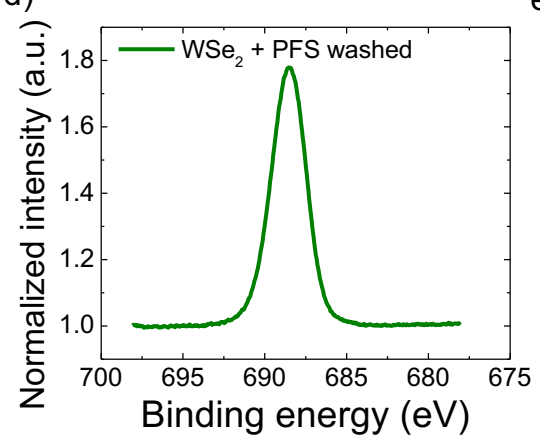

g)

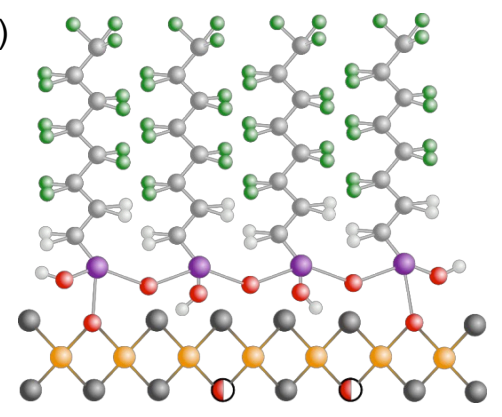

b)

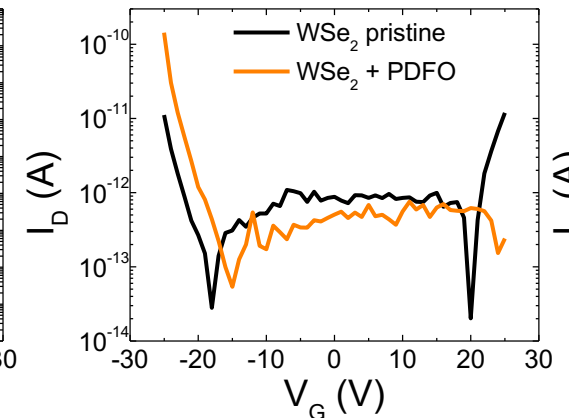

e)

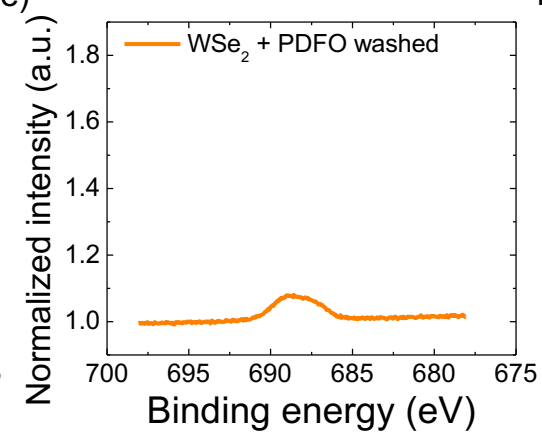

h)

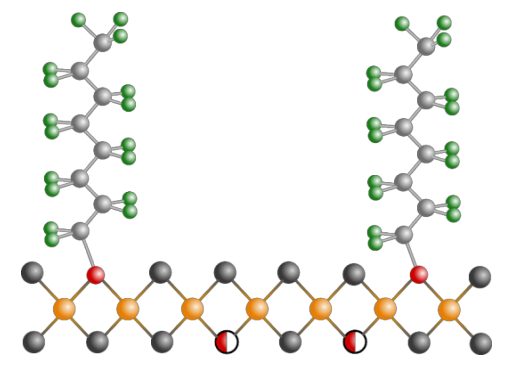

c)

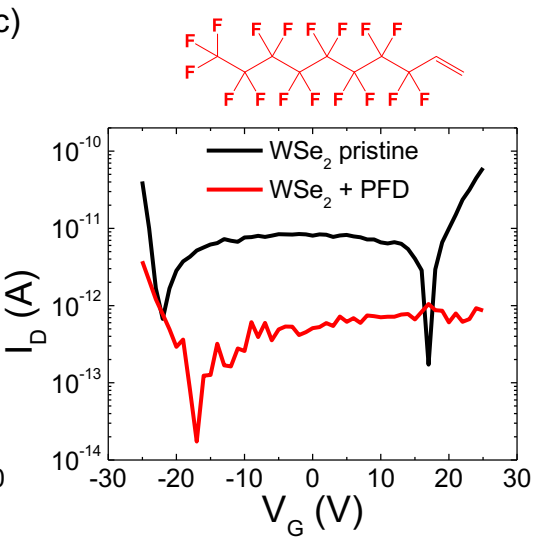

f)

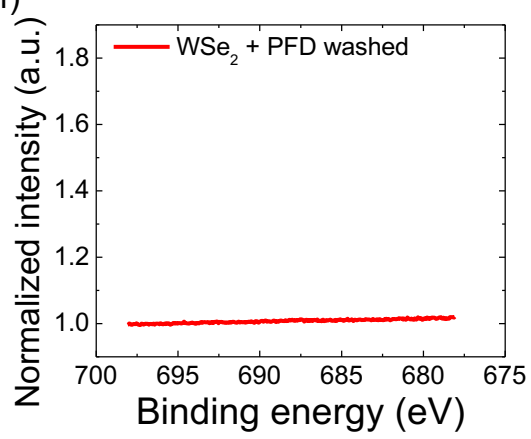

i)

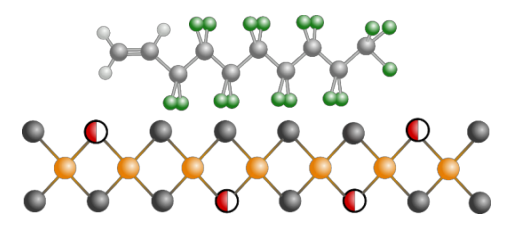

OSe OW OSe vacancy or O substitutional atom OSi OC $\odot \mathrm{O}$ o $\circ \mathrm{H}$

Figure 4. (a- c) Transfer curves of pristine $\mathrm{WSe}_{2}$ single layers on untreated $\mathrm{SiO}_{2}(90 \mathrm{~nm}$ thick), exposed to (a) PFS, (b) PDFO and (c) PFD vapor. The chemical structures of PFS, PDFO and PFD are shown above. Channel length $\mathrm{L}$ and width $\mathrm{W}$ : (a) $\mathrm{L}=6.6 \mu \mathrm{m}$; W=2.4 $\mu \mathrm{m}$; (b) $\mathrm{L}=5.3 \mu \mathrm{m}$; W=1.5 $\mu \mathrm{m}$; (c) $\mathrm{L}=2.4$ $\mu \mathrm{m} ; \mathrm{W}=2.7 \mu \mathrm{m}$. (d-f) F 1s core level spectra of $\mathrm{WSe}_{2}$ bulk crystal after exposure to (d) PFS, (e) PDFO and (f) PFD vapor and subsequent sonication in ethanol and toluene. (g-i) schematic illustration of the proposed anchoring mechanism/adsorption on defective $\mathrm{WSe}_{2}$ (exhibiting vacancies or oxygen substitutional atoms) for each molecule. 
a)
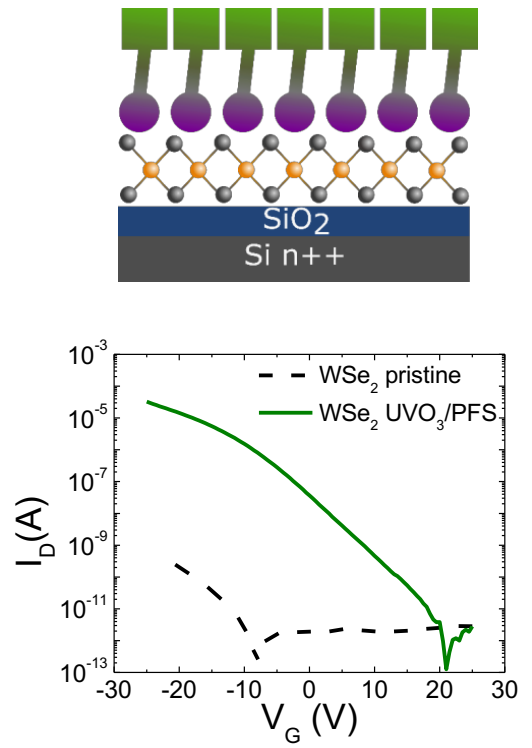

b)
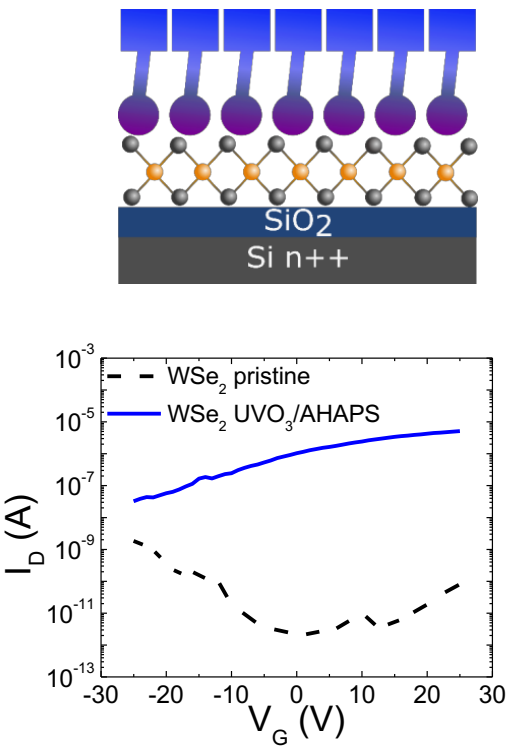

c)
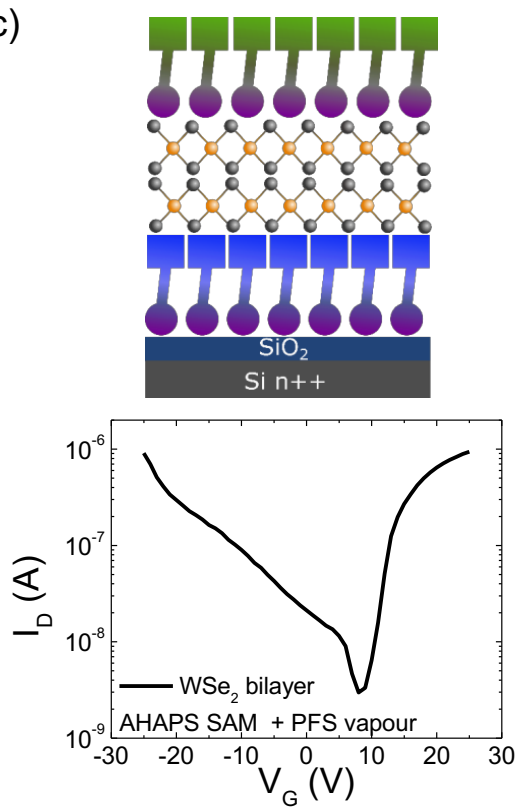

Figure 5. Device architecture and corresponding transfer curves of $(\mathrm{a}, \mathrm{b}) \mathrm{WSe}_{2}$ single layer with $\mathrm{Au}$ contacts, exposed to ozone and (a) PFS vapor and (b) AHAPS vapor. For the sake of comparison, the transfer curves of the pristine $\mathrm{WSe}_{2}$ single layers are also shown (dashed black lines). (c) Device architecture and corresponding transfer curve of bi-layer $\mathrm{WSe}_{2}$ after asymmetric functionalization: the bottom $\mathrm{WSe}_{2}$ layer is in contact with AHAPS-treated $\mathrm{SiO}_{2}$ dielectric, while the top WSe $\mathrm{W}_{2}$ layer was exposed to ozone and PFS vapor. Channel length $\mathrm{L}$ and width $\mathrm{W}$ : (a) $\mathrm{L}=3 \mu \mathrm{m}$; $\mathrm{W}=1.7 \mu \mathrm{m}$; (b) $\mathrm{L}=4.8$ $\mu \mathrm{m} ; \mathrm{W}=2.4 \mu \mathrm{m} ;$ (c) $\mathrm{L}=2.9 \mu \mathrm{m} ; \mathrm{W}=1.2 \mu \mathrm{m}$. 